\title{
An Analytic Route to Tunnelling Splittings Using Semiclassical Perturbation Theory
}

\author{
Timothy A. H. Burd* and David C. Clary* \\ Physical and Theoretical Chemical Laboratory, South Parks Road, Oxford, OX1 3QZ, \\ United Kingdom
}

E-mail: timothy.burd@chem.ox.ac.uk; david.clary@chem.ox.ac.uk 


\begin{abstract}
We present an efficient, analytical and simple route to approximating tunnelling splittings in multidimensional chemical systems, directly from ab initio computations. The method is based on the WKB approximation combined with vibrational perturbation theory. Anharmonicity and corner cutting effects are implicitly accounted for without requiring a full potential energy surface. We test this method on three systems; a model one-dimensional double well potential, the isomerisation of malonaldehyde, and the isomerisation of tropolone. The method is shown to be efficient and reliable.
\end{abstract}

\title{
Graphical TOC Entry
}

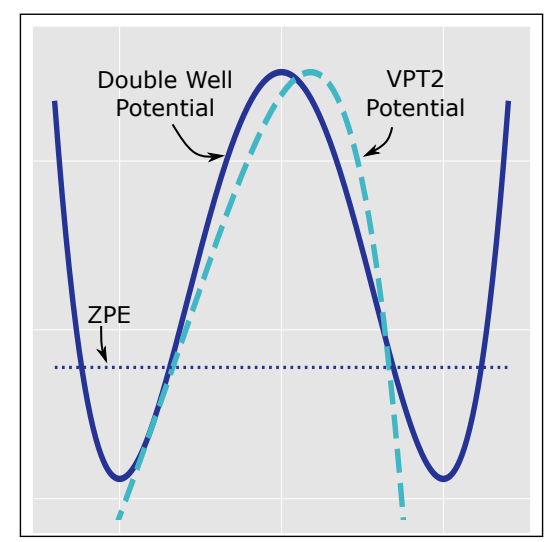

\section{Keywords}

Hydrogen Tunnelling, Semiclassical Transition State Theory, Tunnelling Splitting, Malonaldehyde, Tropolone 


\section{Introduction}

Tunnelling splittings are a spectroscopically observable property of chemical systems where tunnelling between degenerate minima on the potential energy surface is possible. They can be thought of as a result of quantum interactions between two potential wells connected by a tunnelling path; the overlap of two localised vibrational wavefunctions (one in each potential well) leads to symmetric and anti-symmetric eigenstates. These states are separated by an energy $\Delta$, as shown schematically in Fig. 1. These splittings can be measured experimentally ${ }^{1-7}$, and can provide insight into system dynamics, for example in the rearrangement of water clusters ${ }^{8-10}$. The size of the splitting is determined by the extent of overlap of the wavefunctions, and therefore to the ability to tunnel through the potential barrier separating the two wells. Calculations of tunnelling splittings provides a rigorous test of potential energy surfaces and tunnelling theories.

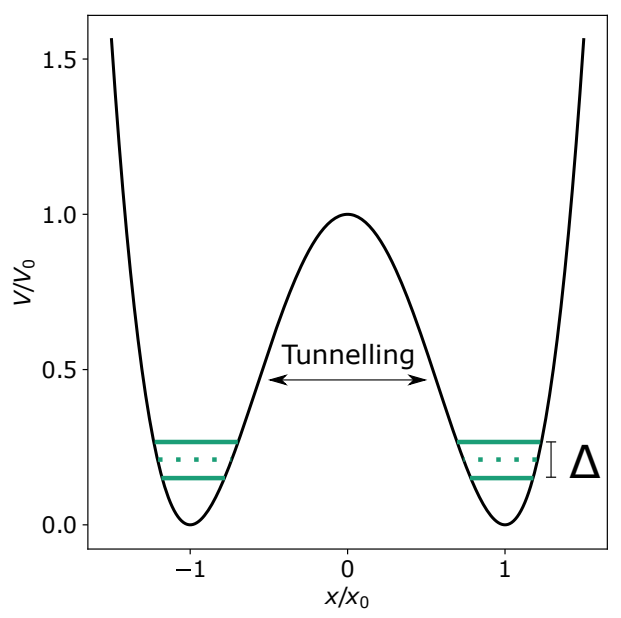

Figure 1: How tunnelling effects the energy levels of a system

A range of methods to determine tunnelling splittings theoretically have been developed. The most accurate (but computationally expensive) of these requires solving the nuclear Schrödinger equation on an accurate multidimensional potential energy surface (PES) using, for example, Diffusion Monte Carlo (DMC) ${ }^{11-14}$, the Multi-configuration Time-dependent 
Hartree (MCTDH) ${ }^{15}$ or other ${ }^{16-20}$ methods. Here the lowest lying vibrational energy levels are accurately determined, which yield $\Delta$ directly. Whilst accurate, this approach requires a full-dimensional PES and expensive dynamical calculations, and so is limited to small systems, for which a PES exists, for example the water dimer ${ }^{21-23}$.

More recently, semiclassical methods based on the path-integral formalism of quantum mechanics have been developed for the calculation of splittings ${ }^{24}$. Instanton methods ${ }^{9,25-29}$ determine a 'dominant' tunneling path by finding the trajectory of least action on the PES, from which $\Delta$ can be found. This still requires a full PES, or many on-the-fly calculations, which may be prohibitively expensive. Since the instanton at zero temperature is required, the instanton is spread out, and a large amount of the potential energy surface is needed.

Much cheaper methods rely on reducing the system to a one-dimensional form, for example by using the minimum energy path ${ }^{30,31}$ or within the sudden approximation ${ }^{32,33}$. Some methods adjust for reaction path curvature effects ${ }^{34-37}$, although this may be a significant expense, involving tens of thousands of ab initio calculations ${ }^{38} . \Delta$ can then be found using the WKB solution to the one-dimensional problem, as derived by Landau and Lipshitz ${ }^{39}$ and others ${ }^{40,41}$, or its slightly alternate form, as derived by Garg $^{42}$.

Reaction path curvature effects must be accounted for (allowing for corner-cutting trajectories), in order to achieve quantitative tunnelling splittings ${ }^{36}$. Accounting for these curvature effects in a systematic, computationally tractable way remains a significant problem, both in reaction kinetics ${ }^{43,44}$ as well as in calculating tunnelling splittings.

Here we present a method to determine approximate tunnelling splittings analytically as a function of the barrier height, vibrational frequencies and anharmonic constants, available from ab initio calculations. This method accounts for reaction path curvature and anhar- 
monic effects, and does not assume the separability of the reaction mode from the other vibrational degrees of freedom. It relies on the WKB approximation, in conjunction with vibrational perturbation theory, and thus is closely related to the Semiclassical Transition State Theory (SCTST) of rate constants, developed by Miller and coworkers ${ }^{45,46}$.

First we will describe the method, and illustrate its application to a model one-dimensional double well potential. The approach is then applied, in full- and reduced-dimensions, to two multidimensional molecular systems - the isomerisation of malonaldehyde and tropolone for which detailed experimental comparisons are possible.

\section{Semiclassical Perturbation Theory of Tunnelling Split-}

\section{tings}

The Semiclassical Perturbation Theory (SPT) method for tunnelling splittings is based on the WKB approximation, in which the energy splitting is given by the Lifshitz-Herring formula ${ }^{39,47}$. This is found by matching the WKB wavefunction in the classically forbidden region to exact harmonic oscillator wavefunctions in the potential wells, or by periodic orbit analysis. It is straightforward to show ${ }^{41}$ that for a double well potential the splitting $\Delta$ for the ground state can be written:

$$
\begin{aligned}
\Delta & =\frac{\omega_{R}}{\pi} \exp \left[-\int_{a}^{b} p(x) d x\right] \\
& \equiv \frac{\omega_{R}}{\pi} \exp \left[-\int_{a}^{b} \sqrt{2 m\left(V(x)-E_{0}\right)} d x\right] \\
& \equiv \frac{\omega_{R}}{\pi} \exp \left[-\theta\left(E_{0}\right)\right]
\end{aligned}
$$

where $\theta\left(E_{0}\right)$ is the semiclassical penetration integral evaluated at energy $E_{0}$, the zero point energy of the reactant, and $V(x)$ is the adiabatic barrier potential. $a$ and $b$ are the 
classical turning points of the potential at energy $E_{0}$, and $\omega_{R}$ corresponds to the reaction mode frequency in the reactant state, identified as the vibrational mode of lowest-frequency that is of the same symmetry as the imaginary mode at the transition state ${ }^{30,31}$.

SPT provides a way to determine $\theta(E)$ analytically, without assuming the separability of the Hamiltonian such that anharmonicicty and corner-cutting effects are accounted for ${ }^{45,48}$. The derivation follows that of Miller et al. ${ }^{46,49}$. The Bohr-Sommerfeld theory is used to relate the $F^{\text {th }}$ action variable of a quantum system to the phase integral of the momentum $p_{F}$ :

$$
2 \pi \hbar\left(n_{F}+0.5\right)=\oint p_{F} \mathrm{~d} q_{F}
$$

The momentum can then be written in terms of the total energy and the effective potential for some vibrational configuration, $\{n\}^{\prime}$, of the transition state,

$$
p_{F}=\left[2 m\left(E_{v}-V_{\{n\}^{\prime}}\left(q_{F}\right)\right)\right]^{1 / 2} .
$$

Thus the phase integral can be shown to be proportional to the barrier penetration integral $\theta_{\{n\}^{\prime}}\left(E_{v}\right)$, where the $F^{t h}$ degree of freedom corresponds to the reaction mode in a transition state:

$$
n_{F}+0.5=\frac{i}{\pi} \theta_{\{n\}^{\prime}}\left(E_{v}\right)
$$

Second order vibrational perturbation theory (VPT2) ${ }^{46}$ is then used to relate the total energy to the $\mathrm{F}^{\text {th }}$ action variable: 


$$
E_{\{n\}}=\sum_{i=1}^{F} \hbar \omega_{i}\left(n_{i}+0.5\right)+\sum_{i \leq j}^{F} x_{i j}\left(n_{i}+0.5\right)\left(n_{j}+0.5\right)+G_{0} .
$$

Inserting equation 4 into equation 5 , and solving the quadratic equation in $\theta_{\{n\}^{\prime}}\left(E_{v}\right)$ gives:

$$
\theta_{\{n\}^{\prime}}\left(E_{v}\right)=\pi \frac{-\Omega_{\{n\}^{\prime}}+\left[\Omega_{\{n\}^{\prime}}^{2}+4 x_{F F}\left(\Delta V_{f}+G_{0}-E_{\{n\}^{\prime}}-E_{v}\right)\right]^{1 / 2}}{2 x_{F F}}
$$

where

$$
\Omega_{\{n\}^{\prime}}=\frac{\hbar \omega_{F}}{i}+\sum_{i=1}^{F-1} \frac{x_{i F}}{i}\left(n_{i}+0.5\right)
$$

and

$$
E_{\{n\}^{\prime}}=\sum_{i=1}^{F-1} \hbar \omega_{i}\left(n_{i}+0.5\right)+\sum_{i \leq j}^{F-1} x_{i j}\left(n_{i}+0.5\right)\left(n_{j}+0.5\right)+G_{0}
$$

The VPT2 constants, $x_{i j}$, are calculated using the third and semidiagonal fourth order derivatives of the potential along the normal modes, $f_{i j k}$ and $f_{i i j j}$ respectively ${ }^{46}$. These higher order derivatives can be obtained from an analytically fitted PES ${ }^{50,51}$ or from $a b$ initio calculations ${ }^{52-55}$. $\Delta$ can then be calculated using equations 1 and 6 . Anharmonic ZPE effects are also accounted for in the reactant well via perturbation theory.

Note that higher order perturbation theory treatments, such as VPT4, are possible ${ }^{56}$. In the VPT4 theory, $\theta_{\{n\}^{\prime}}\left(E_{v}\right)$ is found as the root of a cubic equation, which can be found numerically for each energy, $E_{v}$. This procedure also requires higher order derivatives of the PES and so is too costly for most chemical applications. Similarly, lower order treatments are possible. VPT0 is equivalent to the parabolic barrier approximation, and so only the 
barrier height and harmonic frequencies are required. In this case, the formula becomes:

$$
\theta_{\{n\}^{\prime}}\left(E_{v}\right)=i \pi \frac{E_{v}-E_{\{n\}^{\prime}}^{0}}{\omega_{F}}
$$

with

$$
E_{\{n\}^{\prime}}^{0}=\sum_{i=1}^{F-1} \hbar \omega_{i}\left(n_{i}+0.5\right) .
$$

For systems where calculating the full anharmonic matrix is too expensive, a one-dimensional form of VPT2-SPT exists ${ }^{51,57,58}$ in which the reaction mode is treated as separable from the other (bound) degrees of freedom. In this case, only the $x_{F F}$ term of the matrix need be evaluated, in its approximate one-dimensional form ${ }^{48}$ :

$$
x_{i j}= \begin{cases}x_{F F}^{1 D} & \text { if } i=j=F \\ 0 & \text { otherwise }\end{cases}
$$

This 1D method is well tested in kinetics ${ }^{51,58}$ and can be an efficient route to accurate rate constants.

In this work we calculate splittings through the vibrational ground state of the TS, and study systems with just two degenerate minima. It is relatively straightforward to extend this to systems with multiple degenerate minima, connected by various transition states (for example, in water clusters), for example using a Hückel-type approximation ${ }^{9,30,31}$. An extension of the semiclassical formula for tunnelling splittings in vibrationally excited states is also available $\mathrm{e}^{42,59}$, but is not studied further in this work. 


\section{Quartic Double Well Potential}

We can gain insight into the accuracy of SPT, by comparison to known exact quantum and instanton results for a one-dimensional double well potential of the form:

$$
V(x)=V_{0}\left(\frac{x^{2}}{x_{0}^{2}}-1\right)^{2}
$$

SPT parameters for this potential can be calculated analytically. Since higher order derivatives are easy to obtain for this potential, we can also determine the impact of the level of vibrational perturbation theory used. SPT results using VPT0, VPT2 and VPT4 were calculated for this potential with a range of barrier heights, $V_{0}$. $x_{0}$ was chosen such that the harmonic ZPE remains constant between them:

$$
x_{0}=5 \sqrt{V_{0}} \Longrightarrow \text { Harmonic } \mathrm{ZPE} \approx 0.283
$$

Equation 1 can also be solved numerically, for the double well potential, with $E_{0}$ taken as the harmonic ZPE, giving the instanton result. Comparison between the SPT and instanton results shows errors in the VPTn barrier approximation, as well as differences in the treatment of anharmonicity in the reactant well. Comparison of the instanton to quantum results indicates the errors in the Lifshitz-Herring formula itself. Table 1 and Fig. 2 compare SPT results with instanton and exact quantum results calculated by exact diagonalisation of the Hessian, using a particle-in-a-box wavefunction basis.

It is clear, from comparison of the instanton and quantum results, that the LifshitzHerring formula is excellent in the case of the large barriers, however it overestimates the splitting when the ZPE is close to the barrier top; it differs from the exact quantum result for $V_{0}=0.25$ by nearly a factor of two. This is caused by the harmonic treatment of the well region in the derivation of equation 1, which is a poor approximation when the ZPE is close to (or above) the barrier top. For small barriers, the SPT method performs better 
than the instanton method, owing to the anharmonic treatment of the ZPE in SPT . For the barriers where the ZPE lies well below the barrier top, the Lifshitz-Herring formula remains an excellent approximation.

The SPT method behaves similarly to the instanton method for small barriers, again due to the breakdown of the Lifshitz-Herring formula. For larger barriers, however, the perturbation theory approximation itself performs less well as $V_{0}$ increases. This is indicated by the deviation of the VPTn results from the instanton values. For the larger barriers, the ZPE lies well below the barrier top, and so the barrier is less well described by the derivatives of the PES at the TS.

Table 1: $\Delta$ values for the double well potential with varying $V_{0}$ and $x_{0}=5 \sqrt{V_{0}}$. Instanton and exact quantum solutions are also included. Powers of ten are in parentheses.

\begin{tabular}{|c|ccc|cc|}
\hline$V_{0}$ & VPT0 & VPT2 & VPT4 & Instanton & Quantum \\
\hline 2 & $2.50(-7)$ & $6.62(-8)$ & $4.92(-8)$ & $4.17(-8)$ & $4.15(-8)$ \\
1 & $6.45(-4)$ & $3.96(-4)$ & $3.59(-4)$ & $3.73(-4)$ & $3.42(-4)$ \\
0.5 & $3.27(-2)$ & $2.78(-2)$ & $2.66(-2)$ & $3.00(-2)$ & $2.25(-2)$ \\
0.25 & $2.33(-1)$ & $1.99(-1)$ & $1.88(-1)$ & $2.32(-1)$ & $1.19(-1)$ \\
\hline
\end{tabular}

It is clear that overall the VPT2-SPT performs well except for very high barriers; for $V_{0}=1$, the VPT2 result differs from the instanton result by just $6 \%$. The success of VPT2SPT can be understood by visualising the VPT2 barriers. Since, VPT2-SPT gives the exact value of $\theta(E)$ for an inverted Morse barrier ${ }^{48}$, this can illustrate the 'effective barrier' used in the calculation. These are shown in Fig. 3 for the barriers in Table 1. The VPT2 effective barrier clearly provides a good description of the model potential in the tunnelling region, and is able to accurately predict the barrier width at the ZPE. Increasing the level of perturbation theory will give an even better fit. 


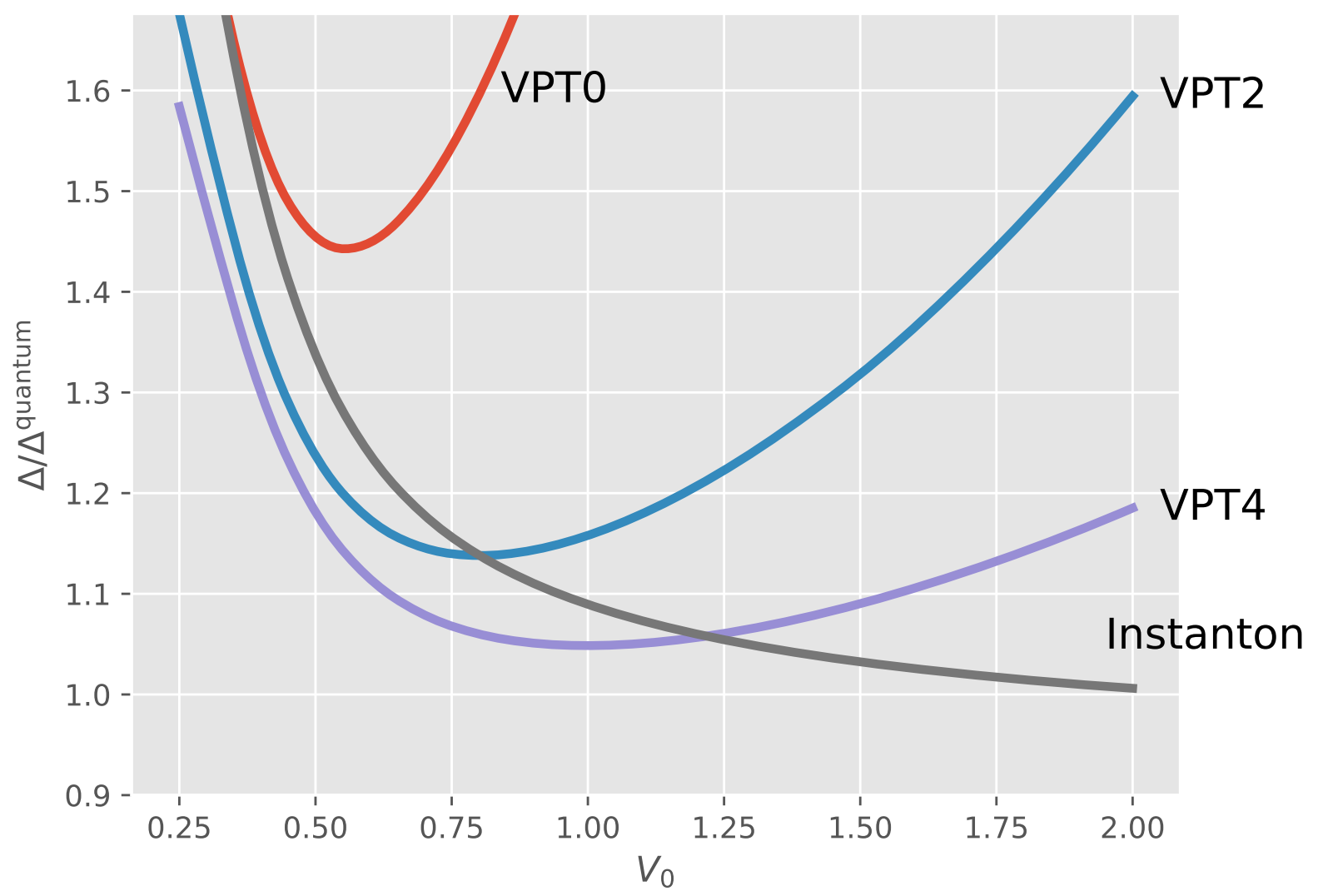

Figure 2: Comparison of the SPT and instanton results with exact quantum tunnelling splittings for the quartic double well potential. 


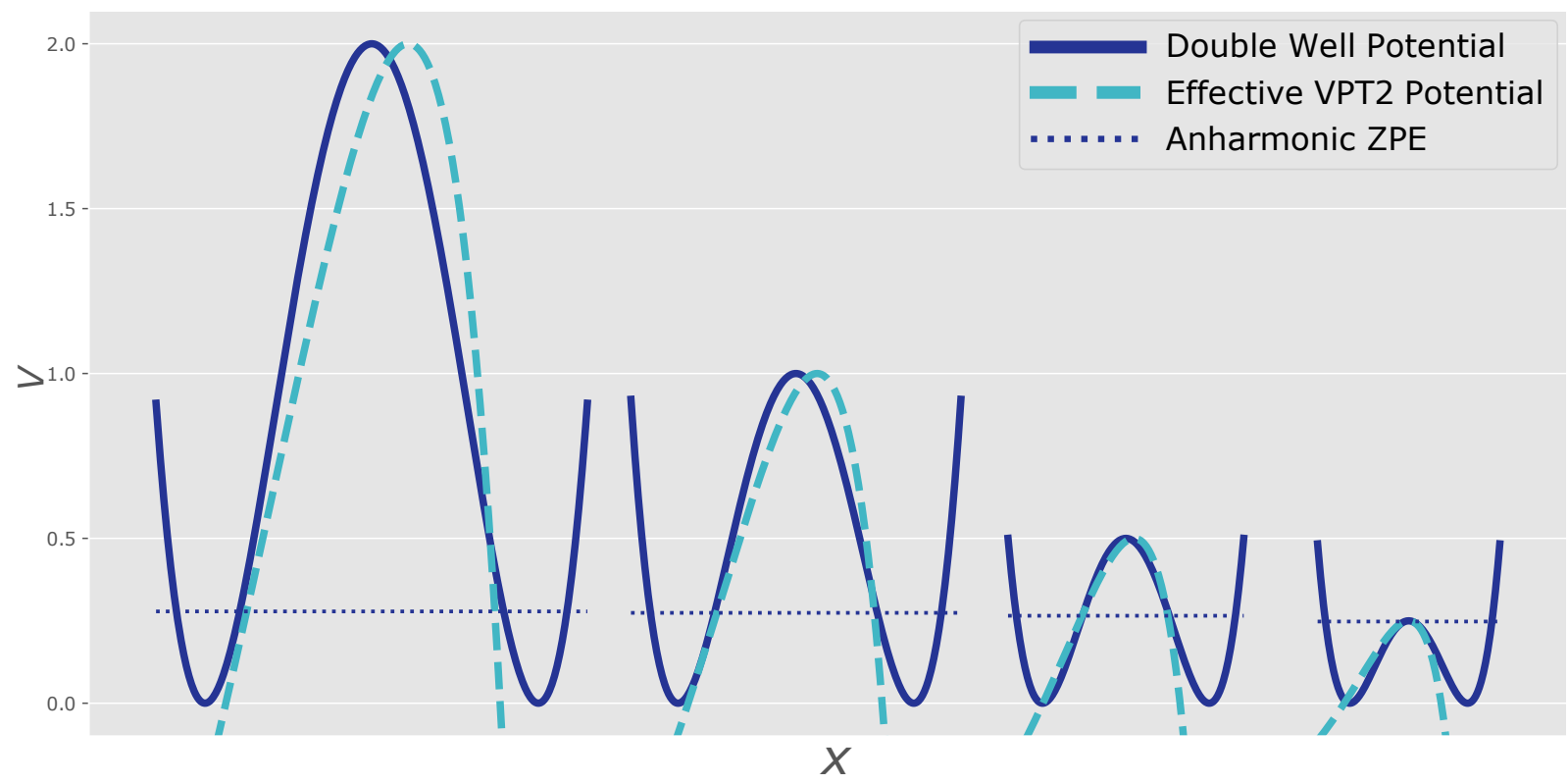

Figure 3: The effective VPT2-SPT well potential for the wells in Table 1

It is clear the VPT0 treatment is insufficient to give quantitative splittings. It is a factor of six larger than the instanton splitting for the $V_{0}=2$ barrier. The effective parabolic barrier is significantly narrower, in general, than the double well potential, so the splitting is overestimated. VPT4, whilst too expensive for most chemical applications, performs very well, reproducing the instanton result to great accuracy over the whole range of barrier heights.

Note that the error due to the perturbation theory approximation is very sensitive to the exact form of the model potential used. It is thus not easy to precisely quantify this error for real, multidimensional reaction potentials. These tests, however, indicate VPT2 is likely to be sufficiently accurate to be useful for these purposes. It is possible that a deep tunnelling correction, analogous to that of Wagner for bimolecular reactive systems ${ }^{60}$, may be able to further improve these results for large barriers. 
A Mathematica ${ }^{61}$ notebook reproducing the SPT results for the double well potential is available in the supplementary material.

\section{Application to Malonaldehyde}

We now test VPT2-SPT for a real chemical system; the isomerisation of Malonaldehyde. Malonaldehyde shows a tunnelling splitting through an intramolecular H-transfer, as shown

in Fig. 4. The splitting has been investigated experimentally ${ }^{4-6}$ and theoretically ${ }^{13,15,62-66}$, and is a common test case for tunnelling splitting theories.

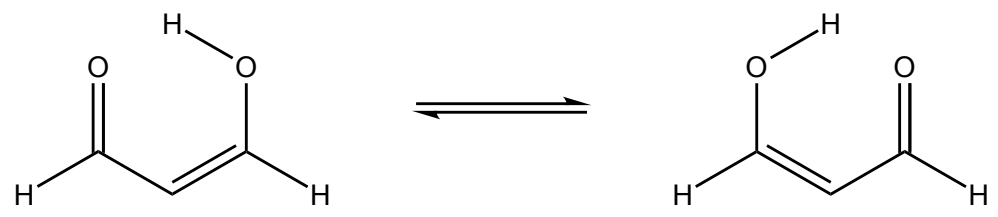

Figure 4: Isomerisation of malonaldehyde

\section{Computational Methods}

We performed ab initio calculations on Malonaldehyde using Gaussian $09^{67}$. VPT2-SPT calculations were performed using our in-house pySCTST $\operatorname{code}^{68}$. Molecular structures were optimised, and frequencies calculated at the MP2 level of theory, and barrier heights were corrected using $\operatorname{CCSD}(\mathrm{T})$ calculations at the MP2 optimised geometry. A tight convergence criterion was used in the optimisation step. Anharmonic constants were determined using the freq=anharmonic keyword in Gaussian. In the 1D calculations, 8 single point MP2 energies were calculated along the reaction mode, away from the TS, with a step size of $\delta Q=0.02 a_{0} \mathrm{Da}^{0.5}$ in order to determine the anharmonicity of the reaction path, calculated using Richardson extrapolation. 
Calculations were performed using the cc-pVTZ, and the aug-cc-pVQZ basis sets ${ }^{69}$, to determine the sensitivity of these calculations to basis set size. For the aug-cc-pVQZ calculations, anharmonic constants from the cc-pVTZ calculations were used, due to the prohibitive expense of using the larger basis set. This method of using a cheaper method to determine just the anharmonic constants has been previously tested successfully ${ }^{70}$.

\section{Results}

The SPT parameters found from the ab initio calculations are shown in Table 2, for both the isomerisation shown in Fig. 4, and for the deuterium-substituted isomer. By comparing one-dimensional (1D) and full-dimensional (FD) results, the effect of corner cutting through the potential energy surface is clear. Including all degrees of freedom in the anharmonic calculation leads to a larger effective barrier height, and a less negative anharmonic constant (corresponding to a narrower barrier). The effective imaginary frequency, $\Omega_{\{0\}}$, itself is relatively unchanged, since the effect of the $x_{i F}$ s largely cancel out (see Fig. 5).

Table 2: Adiabatic barrier heights, effective imaginary frequencies and anharmonic constants for aug-cc-pVQZ levels of theory for malonaldehyde.

\begin{tabular}{|l|ccccc|}
\hline & SPT Level & $V_{0}(\mathrm{~kJ} / \mathrm{mol})$ & $\Omega_{\{0\}}\left(\mathrm{cm}^{-1}\right)$ & $x_{F F}\left(\mathrm{~cm}^{-1}\right)$ & $\omega_{R}\left(\mathrm{~cm}^{-1}\right)$ \\
\hline \hline \multirow{2}{*}{ H-transfer } & 1D & 5.64 & 1185 & -319 & 290 \\
& FD & 6.37 & 1187 & -274 & 290 \\
\hline \multirow{2}{*}{ D-transfer } & 1D & 9.28 & 887 & -190 & 280 \\
& FD & 10.0 & 873 & -145 & 280 \\
\hline
\end{tabular}

Table 3 shows good agreement between SPT and experiment. It is clear by comparing cc-pVTZ and aug-cc-pVQZ results that using high quality ab initio calculations is essential. This is straightforward in SPT due to the relatively few calculations required compared to other methods. The MCTDH, DMC and instanton calculations were all performed in full 
Table 3: $\Delta$ values for Malonaldehyde from theory and experiment. Values in bold are from this work.

\begin{tabular}{|c|c|c|c|}
\hline & Method & Reference & $\Delta / \mathrm{cm}^{-1}$ \\
\hline \multirow{10}{*}{ 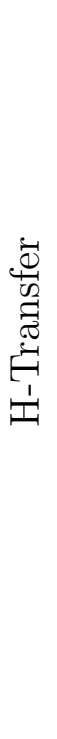 } & 1D SPT (cc-pVTZ) & & 26.48 \\
\hline & 1D SPT (aug-cc-pVQZ) & & 22.23 \\
\hline & FD SPT (cc-pVTZ) & & 21.69 \\
\hline & FD SPT (aug-cc-pVQZ) & & 18.69 \\
\hline & MCTDH (CCSD $(\mathrm{T})$ based PES) & Schröder, $2011^{15}$ & 23.4 \\
\hline & DMC (CCSD $(\mathrm{T})$ based PES) & Wang, $2008^{14}$ & $21.6 \pm 2$ \\
\hline & Instanton $(\mathrm{CCSD}(\mathrm{T})$ based PES) & Cvitaš, $2016^{63}$ & 25 \\
\hline & Experiment & Baughcum, $1981^{6}$ & 21.58 \\
\hline & Experiment & Firth, $1991^{5}$ & 21.6 \\
\hline & Experiment & Baba, $1999^{4}$ & 21.58 \\
\hline \multirow{7}{*}{ 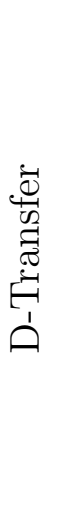 } & 1D SPT (cc-pVTZ) & & 3.33 \\
\hline & 1D SPT (aug-cc-pVQZ) & & 2.267 \\
\hline & FD SPT (cc-pVTZ) & & 2.694 \\
\hline & FD SPT (aug-cc-pVQZ) & & 2.065 \\
\hline & Instanton $(\mathrm{CCSD}(\mathrm{T})$ based PES) & Cvitaš, $2016^{63}$ & 3.4 \\
\hline & DMC (CCSD $(\mathrm{T})$ based PES) & Wang, $2008^{14}$ & $3.0 \pm 2$ \\
\hline & Experiment & Baughcum, $1984^{6}$ & 2.915 \\
\hline
\end{tabular}




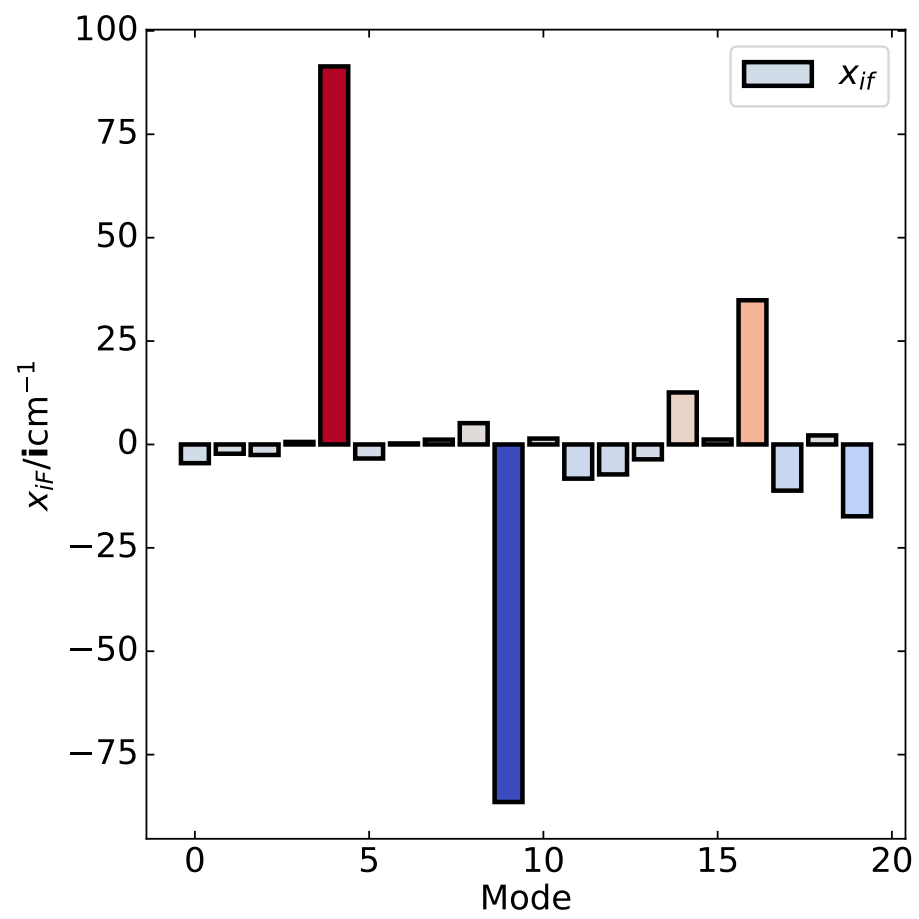

Figure 5: Values for $x_{i F}$ for malonaldehyde at the cc-pVTZ level of theory. Bar colours reflect their values. Modes labeled in order of descending frequency.

dimensions on the same surface by Wang et $a l^{14}$, fit to 11,147 near basis-set-limit frozencore $\operatorname{CCSD}(\mathrm{T})$ electronic energies. SPT agrees within $15 \%$ of the experimental value for the H-transfer, and within $30 \%$ for the deuterium transfer. This is similar to the errors obtained by using different ab initio potentials; for example the augmented and non-augmented basis set results differ by about $30 \%$.

\section{Application to Tropolone}

Tropolone also presents a tunnelling splitting, although much smaller than that of malonaldehyde, due to the larger barrier to isomerisation. The tropolone motif is found in many natural products, and has known biological activity ${ }^{71}$, so has been the subject of experimen$\operatorname{tal}^{7,72}$ and theoretical ${ }^{32,33,37,73}$ interest. Its structure is shown in Fig. 6. For this system, 
with 15 atoms, a full-dimensional treatment using a large basis set is prohibitively expensive. Here we present results of using SPT in one dimension, as well as the ' $1 \mathrm{D}+$ ' formalism developed for SCTST in previous work ${ }^{74}$. Here, a 1D SPT calculation is performed, but the full-dimensional value of $x_{F F}$ is used. Thus vibrational couplings are partially accounted for, and a better estimate of the shape of the barrier is used. The computational cost is just two Hessian calculations more than 1D SPT, and has been shown to be a useful correction to 1D-SCTST in kinetics ${ }^{70,74}$.
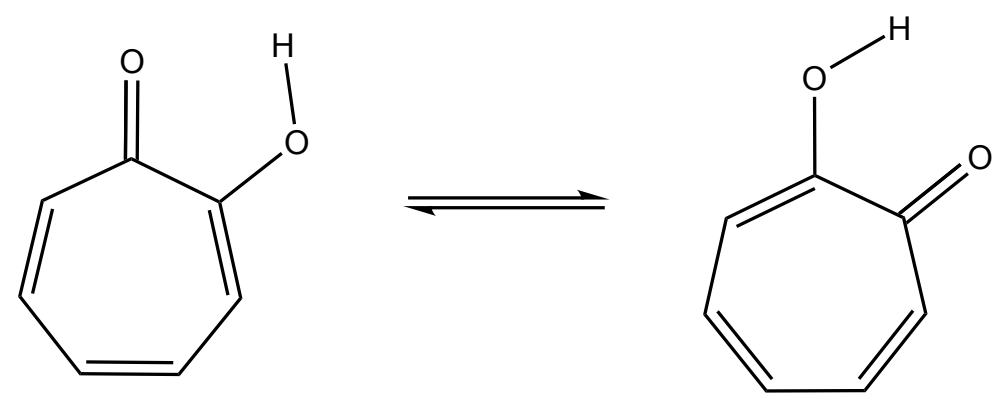

Figure 6: Isomerisation of Tropolone

SPT parameters are shown in Table 4 and the resultant splittings are shown in Table 5. 1D and $1 \mathrm{D}+\mathrm{SPT}$ perform very well for the H-transfer reaction, both lying within $8 \%$ of the experimental values. Again we find the agreement is less close for deuterium transfer; the $1 \mathrm{D}+$ estimates a splitting about half as large as experiment, likely due to the errors in approximating the larger barrier shape with derivatives at the TS.

\section{An anharmonic Lifshitz-Herring formula}

It is clear from Fig. 2 that the breakdown of the harmonic well approximation can have significant impact on the accuracy of the Lifshitz-Herring formula method when the ZPE is near the barrier top. Whilst the anharmonic ZPE of the reactant well is taken into account in the SPT method described, the form of the prefactor in equation 1 is, itself, based on a 
Table 4: Adiabatic barrier heights, effective imaginary frequencies and anharmonic constants for aug-cc-pVQZ levels of theory studied in this work for the isomerisation of Tropolone

\begin{tabular}{|c|ccccc|}
\hline & SPT Level & $V^{f}(\mathrm{~kJ} / \mathrm{mol})$ & $\Omega_{F}\left(i \mathrm{~cm}^{-1}\right)$ & $x_{F F}\left(\mathrm{~cm}^{-1}\right)$ & $\omega_{R}\left(\mathrm{~cm}^{-1}\right)$ \\
\hline \hline \multirow{3}{*}{ H-transfer } & 1D & 17.22 & 1231 & -190 & 350 \\
& $1 \mathrm{D}+$ & 17.22 & 1231 & -183 & 350 \\
\hline \multirow{3}{*}{ D-transfer } & 1D & 20.82 & 906.2 & -24.1 & 336 \\
& $1 D+$ & 20.82 & 906.2 & -96.2 & 336 \\
\hline
\end{tabular}

Table 5: $\Delta$ values for Tropolone from theory and experiment. Values in bold are from this work.

\begin{tabular}{|c|c|c|c|}
\hline & Method & Reference & $\Delta / \mathrm{cm}^{-1}$ \\
\hline \multirow{5}{*}{ 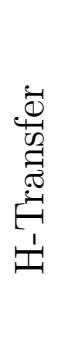 } & 1D SPT (aug-cc-pVQZ) & & 0.904 \\
\hline & 1D+ SPT (aug-cc-pVQZ) & & 0.978 \\
\hline & Experiment & Tanaka, $1999^{7}$ & 0.974 \\
\hline & Experiment & Frost, $1996^{75}$ & $1.0 \pm 0.4$ \\
\hline & Experiment & Redington, $2008^{76}$ & 0.974 \\
\hline \multirow{3}{*}{ 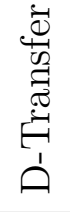 } & 1D SPT (aug-cc-pVQZ) & & 0.181 \\
\hline & 1D+ SPT (aug-cc-pVQZ) & & 0.023 \\
\hline & Experiment & Redington, $2008^{76}$ & 0.051 \\
\hline
\end{tabular}


harmonic well approximation. An anharmonic description of the well may therefore improve the method in this regime. In SPT, a full anharmonic calculation of the reactant well is already required in order to correct the ZPE to its anharmonic value, therefore an improved well description does not require any additional $a b$ initio calculations.

Following the derivation of Miller ${ }^{41}$, the prefactor can be improved by, for example, using an anharmonic estimation of the quantised energy levels in the well:

$$
E(n)=\omega_{R}\left(n+\frac{1}{2}\right)+x_{R R}\left(n+\frac{1}{2}\right)^{2}
$$

which gives:

$$
\begin{aligned}
n(E) & =\frac{-\omega_{R} \pm \sqrt{\omega^{2}+4 E x_{R R}}}{2 x}-\frac{1}{2} \\
n^{\prime}(E) & =\frac{1}{\omega_{R}} \frac{1}{\sqrt{1-\frac{E}{D}}}
\end{aligned}
$$

where:

$$
D=-\frac{\omega_{R}^{2}}{4 x_{R R}}
$$

The improved splitting formula is now given by a simple adaption of equation 1:

$$
\Delta^{\text {anharm }}=\frac{\omega_{R}}{\pi} \sqrt{1-\frac{E}{D}} \exp \left[-\theta\left(E_{0}\right)\right]
$$

In the harmonic limit, $x_{R R} \rightarrow 0$, the harmonic result is returned. We can test this improved model on the model double well potential (equations 12 and 13), with the results shown in Fig. 7. Clearly this offers a significant improvement for small barriers, and a small 
improvement for large barriers, as expected. It performs significantly better than the instanton method (which relies on a harmonic well approximation) in the small barrier regime.

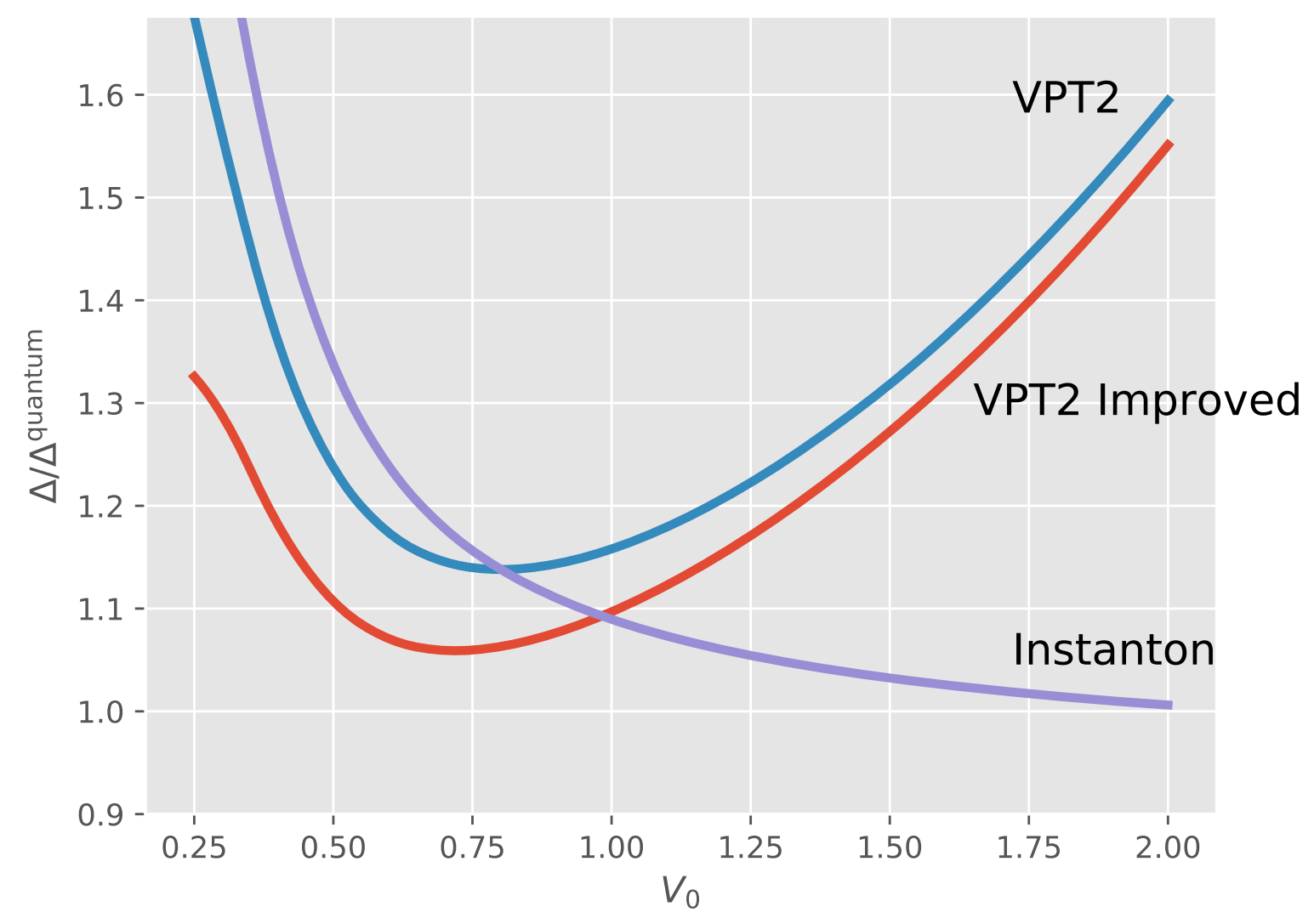

Figure 7: Improving the description of the well region gives an improved splitting, particularly when the ZPE is near the barrier top

\section{Conclusions}

We have found that the WKB approximation, in combination with vibrational perturbation theory, allows for a simple, cost-effective and analytical route to tunnelling splittings in multidimensional systems, that strongly resembles the SCTST theory of reaction rates. This Semiclassical Perturbation Theory method accounts for anharmonic and corner-cutting 
effects by including couplings between vibrational modes. It can also be systematically improved with higher orders of perturbation theory, although the expense is likely prohibitive for calculations going beyond second order. The SPT method for splittings can also take advantage of the various approximations that have been developed for the SCTST theory of rates, such as reduced-dimensional methods.

Testing on a model one-dimensional potential indicates that SPT is a good approximation to the exact result for a range of barrier heights. The semiclassical approximation itself is, as expected, most accurate when applied to systems with a high and wide barrier. The method's application (in full and reduced dimensions) to multidimensional systems was illustrated by application to Malonaldehyde and Tropolone splittings. In both cases SPT performed well, with calculated splittings lying close to experimental results and other theoretical calculations. We also illustrate that an accurate theoretical result is highly dependent on high quality $a b$ initio calculations, further motivating the need for theories that require an absolute minimum of such computations. Variants of the SPT method, such as the 1D and 1D+ methods, may satisfy these needs.

Since the method does not require a PES, and only a few ab initio computations are needed, this method could have application in approximating tunnelling splittings in large and complex systems for which a full treatment of the dynamics may not be possible.

\section{Supplementary Material}

A Mathematica ${ }^{61}$ notebook reproducing the SPT results for the model double well potential is available in the supplementary material. This information is available free of charge via the Internet at http://pubs.acs.org. 


\section{Acknowledgements}

T. A. H. Burd is grateful for support from the EPSRC Centre for Doctoral Training in Theory and Modelling in Chemical Sciences (Project Grant No. EP/L015722/1). The authors also thank Karl-Michael Ziems for helpful discussions.

\section{References}

(1) Goroya, K. G.; Zhu, Y.; Sun, P.; Duan, C. High Resolution Jet-Cooled Infrared Absorption Spectra of the Formic Acid Dimer: A Reinvestigation of the C-O Stretch Region. J. Chem. Phys. 2014, 140, 164311.

(2) Ortlieb, M.; Havenith, M. Proton Transfer in $(\mathrm{HCOOH})_{2}$ : An IR High-Resolution Spectroscopic Study of the Antisymmetric C-O Stretch. J. Phys. Chem. A 2007, 111, $7355-7363$.

(3) Baughcum, S. L.; Duerst, R. W.; Rowe, W. F.; Smith, Z.; Wilson, E. B. Microwave Spectroscopic Study of Malonaldehyde (3-Hydroxy-2-Propenal). 2. Structure, Dipole Moment, and Tunneling. J. Am. Chem. Soc. 1981, 103, 6296-6303.

(4) Baba, T.; Tanaka, T.; Morino, I.; Yamada, K. M. T.; Tanaka, K. Detection of the Tunneling-Rotation Transitions of Malonaldehyde in the Submillimeter-Wave Region. J. Chem. Phys. 1999, 110, 4131-4133.

(5) Firth, D. W.; Beyer, K.; Dvorak, M. A.; Reeve, S. W.; Grushow, A.; Leopold, K. R. Tunable Far-infrared Spectroscopy of Malonaldehyde. J. Chem. Phys. 1991, 94, 18121819.

(6) Baughcum, S. L.; Smith, Z.; Wilson, E. B.; Duerst, R. W. Microwave Spectroscopic Study of Malonaldehyde. 3. Vibration-Rotation Interaction and One-Dimensional Model for Proton Tunneling. J. Am. Chem. Soc. 1984, 106, 2260-2265. 
(7) Tanaka, K.; Honjo, H.; Tanaka, T.; Kohguchi, H.; Ohshima, Y.; Endo, Y. Determination of the Proton Tunneling Splitting of Tropolone in the Ground State by Microwave Spectroscopy. J. Chem. Phys. 1999, 110, 1969-1978.

(8) Richardson, J. O.; Wales, D. J.; Althorpe, S. C.; McLaughlin, R. P.; Viant, M. R.; Shih, O.; Saykally, R. J. Investigation of Terahertz Vibration-Rotation Tunneling Spectra for the Water Octamer. J. Phys. Chem. A 2013, 117, 6960-6966.

(9) Richardson, J. O.; Althorpe, S. C.; Wales, D. J. Instanton Calculations of Tunneling Splittings for Water Dimer and Trimer. J. Chem. Phys. 2011, 135, 124109.

(10) T. Cvitaš, M.; O Richardson, J. Quantum Tunnelling Pathways of the Water Pentamer. Phys. Chem. Chem. Phys. 2019,

(11) Gregory, J. K.; Clary, D. C. Calculations of the Tunneling Splittings in Water Dimer and Trimer Using Diffusion Monte Carlo. J. Chem. Phys. 1995, 102, 7817-7829.

(12) Sorenson, J. M.; Gregory, J. K.; Clary, D. C. Diffusion Monte Carlo Studies of IsotopeSubstituted Water Trimers. Chem. Phys. Lett. 1996, 263, 680-686.

(13) Viel, A.; Coutinho-Neto, M. D.; Manthe, U. The Ground State Tunneling Splitting and the Zero Point Energy of Malonaldehyde: A Quantum Monte Carlo Determination. J. Chem. Phys. 2007, 126, 024308.

(14) Wang, Y.; Braams, B. J.; Bowman, J. M.; Carter, S.; Tew, D. P. Full-dimensional quantum calculations of ground-state tunneling splitting of malonaldehyde using an accurate ab initio potential energy surface. J. Chem. Phys 2008, 128, 224314.

(15) Schröder, M.; Gatti, F.; Meyer, H.-D. Theoretical Studies of the Tunneling Splitting of Malonaldehyde Using the Multiconfiguration Time-Dependent Hartree Approach. J. Chem. Phys. 2011, 134, 234307. 
(16) Althorpe, S. C.; Clary, D. C. Calculation of the Intermolecular Bound States for Water Dimer. J. Chem. Phys. 1994, 101, 3603-3609.

(17) Shida, N.; Barbara, P. F.; Almlöf, J. A Reaction Surface Hamiltonian Treatment of the Double Proton Transfer of Formic Acid Dimer. J. Chem. Phys. 1991, 94, 3633-3643.

(18) Althorpe, S. C.; Clary, D. C. A New Method for Calculating the Rovibrational States of Polyatomics with Application to Water Dimer. J. Chem. Phys. 1995, 102, 4390-4399.

(19) Shida, N.; Barbara, P. F.; Almlöf, J. E. A Theoretical Study of Multidimensional Nuclear Tunneling in Malonaldehyde. J. Chem. Phys. 1989, 91, 4061-4072.

(20) Althorpe, S. C.; Clary, D. C.; Bunker, P. R. Calculation of the Far-Infrared Spectra for $(\mathrm{HF})_{2},(\mathrm{HCl})_{2}$ and $(\mathrm{HBr})_{2}$. Chem. Phys. Lett. 1991, 187, 345-353.

(21) Smit, M. J.; Groenenboom, G. C.; Wormer, P. E.; Van Der Avoird, A.; Bukowski, R.; Szalewicz, K. Vibrations, tunneling, and transition dipole moments in the water dimer. J. Phys. Chem. A 2001, 105, 6212-6225.

(22) Huang, X.; Braams, B. J.; Bowman, J. M.; Kelly, R. E. A.; Tennyson, J.; Groenenboom, G. C.; van der Avoird, A. New Ab Initio Potential Energy Surface and the Vibration-Rotation-Tunneling Levels of $\left(\mathrm{H}_{2} \mathrm{O}\right)_{2}$ and $\left(\mathrm{D}_{2} \mathrm{O}\right)_{2}$. J. Chem. Phys. 2008, $128,034312$.

(23) Leforestier, C.; Szalewicz, K.; van der Avoird, A. Spectra of Water Dimer from a New Ab Initio Potential with Flexible Monomers. J. Chem. Phys. 2012, 137, 014305.

(24) Mátyus, E.; Wales, D. J.; Althorpe, S. C. Quantum Tunneling Splittings from PathIntegral Molecular Dynamics. J. Chem. Phys. 2016, 144, 114108.

(25) Richardson, J. O.; Althorpe, S. C. Ring-Polymer Instanton Method for Calculating Tunneling Splittings. J. Chem. Phys. 2011, 134, 054109. 
(26) Richardson, J. O. Full- and Reduced-Dimensionality Instanton Calculations of the Tunnelling Splitting in the Formic Acid Dimer. Phys. Chem. Chem. Phys. 2017, 19, 966970 .

(27) Smedarchina, Z.; Siebrand, W.; Zgierski, M. Z. An Instanton Approach to Intramolecular Hydrogen Exchange: Tunneling Splittings in Malonaldehyde and the Hydrogenoxalate Anion. J. Chem. Phys. 1995, 103, 5326-5334.

(28) Smedarchina, Z.; Fernandez-Ramos, A.; Siebrand, W. Calculation of the Tunneling Splitting in the Zero-Point Level and CO-Stretch Fundamental of the Formic Acid Dimer. Chem. Phys. Lett. 2004, 395, 339-345.

(29) Yagi, K.; Mil'nikov, G. V.; Taketsugu, T.; Hirao, K.; Nakamura, H. Effect of Out-ofPlane Vibration on the Hydrogen Atom Transfer Reaction in Malonaldehyde. Chem. Phys. Lett. 2004, 397, 435-440.

(30) Watanabe, Y.; Taketsugu, T.; Wales, D. J. An Ab Initio Study of Tunneling Splittings in the Water Dimer. J. Chem. Phys. 2004, 120, 5993-5999.

(31) Takahashi, M.; Watanabe, Y.; Taketsugu, T.; Wales, D. J. An Ab Initio Study of Tunneling Splittings in the Water Trimer. J. Chem. Phys. 2005, 123, 044302.

(32) Takada, S.; Nakamura, H. Effects of Vibrational Excitation on Multidimensional Tunneling: General Study and Proton Tunneling in Tropolone. J. Chem. Phys. 1995, 102, 3977-3992.

(33) Guo, Y.; Sewell, T. D.; Thompson, D. L. Semiclassical Calculations of Tunneling Splitting in Tropolone. J. Phys. Chem. A 1998, 102, 5040-5048.

(34) Tautermann, C. S.; Voegele, A. F.; Liedl, K. R. The Ground-State Tunneling Splitting of Various Carboxylic Acid Dimers. J. Chem. Phys. 2003, 120, 631-637. 
(35) Tautermann, C.; Voegele, A.; Loerting, T.; Liedl, K. An Accurate Semiclassical Method to Predict Ground-State Tunneling Splittings. J. Chem. Phys. 2002, 117, 1967-1974.

(36) Brown, F. B.; Tucker, S. C.; Truhlar, D. G. Semiclassical Reaction-path Methods Applied to Calculate the Tunneling Splitting in Ammonia. J. Chem. Phys. 1985, 83, $4451-4455$.

(37) Paz, J. J.; Moreno, M.; Lluch, J. M. Bidimensional Tunneling Splitting in the $\tilde{A}^{1} B_{2}$ and $\tilde{X}^{1} A_{1}$ States of Tropolone. J. Chem. Phys. 1995, 103, 353-359.

(38) Tautermann, C. S.; Voegele, A. F.; Loerting, T.; Liedl, K. R. The Optimal Tunneling Path for the Proton Transfer in Malonaldehyde. J. Chem. Phys. 2002, 117, 1962-1966.

(39) Landau, L. D.; Lifshitz, E. M. Quantum mechanics: non-relativistic theory; Elsevier, 2013; Vol. 3.

(40) Razavy, M. Quantum theory of tunneling; World Scientific, 2003.

(41) Miller, W. H. Periodic orbit description of tunneling in symmetric and asymmetric double-well potentials. J. Phys. Chem. 1979, 83, 960-963.

(42) Garg, A. Tunnel Splittings for One-Dimensional Potential Wells Revisited. Am. J. Phys. 2000, 68, 430-437.

(43) Skodje, R. T.; Truhlar, D. G.; Garrett, B. C. A General Small-Curvature Approximation for Transition-State-Theory Transmission Coefficients. J. Phys. Chem. 1981, 85, 30193023.

(44) Pérez de Tudela, R.; Suleimanov, Y. V.; Richardson, J. O.; Sáez Rábanos, V.; Green, W. H.; Aoiz, F. J. Stress Test for Quantum Dynamics Approximations: Deep Tunneling in the Muonium Exchange Reaction D $+\mathrm{HMu} \rightarrow \mathrm{DMu}+$ H. J. Phys. Chem. Lett. 2014, 5, 4219-4224. 
(45) Miller, W. H. Semi-Classical Theory for Non-Separable Systems:. Construction of "Good" Action-Angle Variables for Reaction Rate Constants. Faraday Discus. 1977, 62, 40-46.

(46) Miller, W. H.; Hernandez, R.; Handy, N. C.; Jayatilaka, D.; Willetts, A. Ab Initio Calculation of Anharmonic Constants for a Transition State, with Application to Semiclassical Transition State Tunneling Probabilities. Chem. Phys. Lett. 1990, 172, 62-68.

(47) Herring, C. Critique of the Heitler-London Method of Calculating Spin Couplings at Large Distances. Rev. Mod. Phys. 1962, 34, 631-645.

(48) Shan, X.; Burd, T. A. H.; Clary, D. C. New Developments in Semiclassical TransitionState Theory. J. Phys. Chem. A 2019, 123, 4639-4657.

(49) Miller, W. H. Semiclassical Limit of Quantum Mechanical Transition State Theory for Nonseparable Systems. J. Chem. Phys. 1975, 62, 1899-1906.

(50) Greene, S. M.; Shan, X.; Clary, D. C. Reduced-Dimensionality Semiclassical Transition State Theory: Application to Hydrogen Atom Abstraction and Exchange Reactions of Hydrocarbons. J. Phys. Chem. A 2015, 119, 12015-12027.

(51) Greene, S. M.; Shan, X.; Clary, D. C. An Investigation of One- versus Two-Dimensional Semiclassical Transition State Theory for H Atom Abstraction and Exchange Reactions. J. Chem. Phys. 2016, 144, 084113.

(52) Nguyen, T. L.; Barker, J. R. Sums and Densities of Fully Coupled Anharmonic Vibrational States: A Comparison of Three Practical Methods. J. Phys. Chem. A 2010, 114, $3718-3730$.

(53) Nguyen, T. L.; Stanton, J. F.; Barker, J. R. Ab Initio Reaction Rate Constants Computed Using Semiclassical Transition-State Theory: $\mathrm{HO}+\mathrm{H}_{2} \rightarrow \mathrm{H}_{2} \mathrm{O}+\mathrm{H}$ and Isotopologues. J. Phys. Chem. A 2011, 115, 5118-5126. 
(54) Barker, J. R.; Nguyen, T. L.; Stanton, J. F. Kinetic Isotope Effects for $\mathrm{Cl}+\mathrm{CH}_{4} \rightleftharpoons$ $\mathrm{HCl}+\mathrm{CH}_{3}$ Calculated Using Ab Initio Semiclassical Transition State Theory. J. Phys. Chem. A 2012, 116, 6408-6419.

(55) Nguyen, T. L.; Stanton, J. F. High-Level Theoretical Study of the Reaction between Hydroxyl and Ammonia: Accurate Rate Constants from 200 to 2500 K. J. Chem. Phys. 2017, 147, 152704.

(56) Stanton, J. F. Semiclassical Transition-State Theory Based on Fourth-Order Vibrational Perturbation Theory: The Symmetrical Eckart Barrier. J. Phys. Chem. Lett. 2016, 7, 2708-2713.

(57) Greene, S. M.; Shan, X.; Clary, D. C. Rate Constants of Chemical Reactions from Semiclassical Transition State Theory in Full and One Dimension. J. Chem. Phys. 2016, $144,244116$.

(58) Burd, T. A. H.; Shan, X.; Clary, D. C. Tunnelling and the Kinetic Isotope Effect in $\mathrm{CH}_{3}+\mathrm{CH}_{4} \rightarrow \mathrm{CH}_{4}+\mathrm{CH}_{3}$ : An Application of Semiclassical Transition State Theory. Chem. Phys. Lett. 2018, 693, 88-94.

(59) Homayoon, Z.; Bowman, J. M.; Evangelista, F. A. Calculations of Mode-Specific Tunneling of Double-Hydrogen Transfer in Porphycene Agree with and Illuminate Experiment. J. Phys. Chem. Lett. 2014, 5, 2723-2727.

(60) Wagner, A. F. Improved Multidimensional Semiclassical Tunneling Theory. J. Phys. Chem. A 2013, 117, 13089-13100.

(61) Inc., W. R. Mathematica, Version 12.0. https://www.wolfram.com/mathematica, Champaign, IL, 2019.

(62) Richardson, J. O. Ring-Polymer Approaches to Instanton Theory. Thesis, University of Cambridge, 2012. 
(63) Cvitaš, M. T.; Althorpe, S. C. Locating Instantons in Calculations of Tunneling Splittings: The Test Case of Malonaldehyde. J. Chem. Theory Comput. 2016, 12, 787-803.

(64) Mil'nikov, G. V.; Nakamura, H. Practical Implementation of the Instanton Theory for the Ground-State Tunneling Splitting. J. Chem. Phys. 2001, 115, 6881-6897.

(65) Tew, D. P.; Handy, N. C.; Carter, S. The vibrations and tunnelling of malonaldehyde on a Møller-Plesset surface. Molecular Physics 2004, 102, 2217-2226.

(66) Tew, D. P.; Handy, N. C.; Carter, S.; Irle, S.; Bowman, J. The internal coordinate path Hamiltonian; application to methanol and malonaldehyde. Molecular Physics 2003, 101, 3513-3525.

(67) Frisch, M. J.; Trucks, G. W.; Schlegel, H. B.; Scuseria, G. E.; Robb, M. A.; Cheeseman, J. R.; Scalmani, G.; Barone, V.; Mennucci, B.; Petersson, G. A. et al. Gaussian09 Revision E.01. Gaussian Inc. Wallingford CT 2009.

(68) Burd, T. pySCTST. https://bitbucket. com/timothyburd/pysctst, 2019.

(69) Dunning, T. H. Gaussian Basis Sets for Use in Correlated Molecular Calculations. I. The Atoms Boron through Neon and Hydrogen. J. Chem. Phys. 1989, 90, 1007-1023.

(70) Burd, T. A. H.; Shan, X.; Clary, D. C. Catalysis and Tunnelling in the Unimolecular Decay of Criegee Intermediates. Phys. Chem. Chem. Phys. 2018, 20, 25224-25234.

(71) Kahn, V.; Andrawis, A. Inhibition of Mushroom Tyrosinase by Tropolone. Phytochemistry 1985, 24, 905-908.

(72) Sekiya, H.; Nagashima, Y.; Nishimura, Y. Electronic Spectra of Jet-cooled Tropolone. Effect of the Vibrational Excitation on the Proton Tunneling Dynamics. J. Chem. Phys. 1990, 92, 5761-5769.

(73) Smedarchina, Z.; Siebrand, W.; Zgierski, M. Z. Mode-specific Hydrogen Tunneling in Tropolone: An Instanton Approach. J. Chem. Phys. 1996, 104, 1203-1212. 
(74) Burd, T. A. H.; Shan, X.; Clary, D. C. Tunnelling in Cyclocarbenes: An Application of Semiclassical Transition State Theory in Reduced Dimensions. Chem. Phys. Lett. 2019, 735, 136783.

(75) Frost, R. K.; Hagemeister, F. C.; Arrington, C. A.; Zwier, T. S.; Jordan, K. D. Fluorescence-dip infrared spectroscopy of tropolone and tropolone-OD. J. Chem. Phys. 1996, 105, 2595-2604.

(76) Redington, R. L.; Redington, T. E.; Sams, R. L. Tunneling Splittings for "O - O Stretching" and Other Vibrations of Tropolone Isotopomers Observed in the Infrared Spectrum Below 800 cm-1. J. Phys. Chem. A 2008, 112, 1480-1492. 